Грушовинчук A.B. / Grushovinchuk A.V.

проф., доктор техн. наук Ковалышин B.B. / prof. Kovalyshyn V.V., Ph.D.

канд. техн. наук Кырылив Я.Б. / Kyryliv Ya.B., Ph.D.

Przyjęty/Accepted/Принята: 11.12.2014;

Zrecenzowany/Reviewed/Рецензирована: 25.05.2015;

Opublikowany/Published/Опубликована: 30.06.2015;

\title{
Обоснование параметров генераторов пены эжекционного типа повышенной огнетушащей эффективности ${ }^{2}$
}

\author{
Identification of Parameters for a Foam Generator with Improved Extinguishing \\ Effectiveness
}

\author{
Określenie parametrów wytwornicy piany typu wyrzutnia o zwiększonej \\ skuteczności gaśniczej
}

\begin{abstract}
АННОТАЦИЯ
Цель: Целью исследования является перемещение в воздухе наклонных гидравлических струй с помощью построения математической модели процесса взаимодействия струй воздушно-механической пены различной кратности.

Методы: Расчеты будут базироваться на интегрировании уравнений движения материальных точек с учетом сопротивления среды. Для этого описано движение струй, направленных под углом, с помощью уравнений динамики брошенных вверх двух или более абсолютно твердых тел, условно связанных между собой силами. При этом положение тел в пространстве в момент времени $\mathrm{t}$ описывается двумя координатами: $\mathrm{x}=\mathrm{x}(\mathrm{t})$ и $\mathrm{y}=\mathrm{y}(\mathrm{t})$.

На основе анализа литературных данных установлено, что наиболее адекватной является квадратичная зависимость силы сопротивления струи при его трении к воздуху. Также принято, что зависимость силы взаимодействия между струями от разности скоростей этих струй характеризуется квадратичной зависимостью.

Результаты: Сначала смоделирован процесс транспортировки струи средней кратности двумя струями низкой кратности. Также определена оптимальная конструктивная схема расположения струй. Установлено влияние ветра на траекторию обобщенной струи.

Итак, схема с нижним расположением струй пены низкой кратности является эффективной. Но при использовании 4 таких струй происходит снижение общей кратности пены комбинированной струи, также повышается расход пенообразователя. Поэтому мы провели сравнительные расчеты для вариантов с нижним размещением трех и двух струй пены низкой кратности. Было установлено, что использование трех струй пены низкой кратности вместо четырех приводит к уменьшению дальности полета лишь на 3\% (0,7 м), но значительно улучшает общую кратность пены обобщенной струи. Дальнейшее уменьшение числа струй (до двух) приводит к более заметному уменьшению эффективности (5,3\%; 1,1 м) и при этом не приводит к улучшению кратности пены обобщенной струи.

Итак, учитывая влияние струй пены низкой кратности на общую кратность пены обобщенной струи, наиболее целесообразной является третья схема расположения с тремя струями - три струи воздушно-механической пены низкой кратности, поддерживают снизу по кругу одну струю пены средней кратности.

Выводы: В данной работе было исследовано взаимодействие струй воздушно-механической пены различной кратности. На основе теоретических и экспериментальных исследований определено оптимальное расположение струй пены, которое позволяет достичь максимального значения дальности подачи пены при минимальных потерях кратности. Результаты данного исследования в дальнейшем будут применены для разработки опытного образца пеногенератора.
\end{abstract}

Ключевые слова: наклонные гидравлические струи, математическая модель, воздушно-механическая пена, уравнения, оптимальная конструктивная схема, кратность пены, дальность подачи пены

Вид статьи: предварительный отчет

\footnotetext{
1 Львовский государственный университет безопасности жизнедеятельности, Украина / Lviv State University of Life Safety, Ukraine / Процентное участие в создание статьи / Percentage contribution: Grushovinchuk A.V. - 50\%, Kovalyshyn V.V. - 25\%; Kyryliv Ya.B. - 25\%; yaroslav_kyryliv@ukr.net

2 Artykuł został wyróżniony przez Komitet Redakcyjny / The article was recognised by the Editorial Committee / Эту статью наградил Редакционный Совет
} 


\section{A BSTRACT}

Aim: The purpose of this research is to examine overhead movements of inclined hydraulic streams of foam and with the aid of a mathematical model illustrate the mutual interaction of mechanical foam streams with variations of expanded foam.

Methods: Calculations are based on differential equations for material movement points with due regard to environmental resistance. With this in mind, the movement of inclined foam streams was described by means of dynamic equations for two or more propelled material bodies which are linked by forces. Wherein the overhead location of bodies, in the time interval $t$, is described by two co-ordinates: $\mathrm{x}=\mathrm{x}(\mathrm{t})$ and $\mathrm{y}=\mathrm{y}(\mathrm{t})$.

Based on an analysis of literature, it was established that an adequate mathematical description is provided by a quadratic function of the resistance force generated by the stream to its friction with air. It was also accepted that dependence of interacting forces between streams and their velocity difference will be described by a quadratic function.

Results: Initially a model was identified, which revealed the delivery process of a medium-expansion foam jet with the use of two streams of low-expansion foam. Additionally, a model with an optimal configuration of the stream system was identified and a description provided, dealing with the influence of wind on the trajectory of a combined jet.

The most effective model turned out to be the one where the low-expansion foam stream is located in the lower position. However, with four such streams there is an overall decrease of expanded foam in the resulting jet and an increase in the consumption of foaming agent. For this reason the authors performed comparative calculations for variations with the location of a lesser number of foam streams. This included the use of three and two streams of low-expansion foam. It was found that a link of three streams of low-expansion foam, instead of four, leads to a reduction in the jet range by only $3 \%(0.7 \mathrm{~m})$, but overall, significantly improves the quality of foaming in the combined jet. A further reduction of streams (to two) results in a noticeable decrease to the range of effectiveness $(5.3 \%, 1.1 \mathrm{~m})$ and does not improve the quality of foaming in the combined jet. In this way, taking account of the influence of low-expansion foam streams on the overall foaming quality, the most logical approach is to use of the third variant of the combination - three streams of low-expansion foam, supporting at the base, an encircled jet generating middle-expansion foam.

Conclusus: During research, tests were performed on the mutual influence of mechanical foam streams with variations of expanded foam. On the basis of experiments, an optimal location of foam streams was specified, which allows the attainment of maximum results (range) in the distribution of foam with minimal losses. Results from tests may be utilised in the future for the development of an experimental foam generating model.

Keywords: inclined hydraulic stream, mathematical model, mechanical foam, equation, optimal construction scheme, multiple foaming, foam delivery range

Type of article: short scientific report

\section{ABSTRAKT}

Cel: Badanie zachodzącego w powietrzu ruchu pochyłych strumieni hydraulicznych piany za pomocą modelu matematycznego oddziaływania wzajemnego strumieni powietrzno-mechanicznej piany o różnej liczbie spienienia.

Metody: Obliczenia będą oparte na rozwiązaniu równań różniczkowych ruchu punktów materialnych z uwzględnieniem oporu środowiska. W tym celu opisano ruch skierowanych pod kątem strumieni piany przy pomocy równań kinematycznych dla wyrzuconych w górę dwóch lub więcej ciał połączonych ze sobą siłami Przy czym położenie ciał w przestrzeni w odstępie czasowym $t$ opisywane jest dwoma współrzędnymi $\mathrm{x}=\mathrm{x}(\mathrm{t})$ oraz $\mathrm{y}=\mathrm{y}(\mathrm{t})$.

Na podstawie analizy danych literaturowych ustalono, że do opisu matematycznego adekwatna jest funkcja kwadratowa siły oporu strumienia do jego tarcia z powietrzem. Przyjęto również, że zależność wzajemnej siły między strumieniami od różnicy ich prędkości opisana będzie funkcją kwadratową.

Wyniki: Na początku przedstawiono model procesu transportu strumienia o średniej liczbie spienienia poprzez dwa strumienie o niskiej liczbie spienienia. Określono również optymalny model układu strumieni. Opisano wpływ wiatru na trajektorię połączonego strumienia. Najdokładniejszy okazał się model, w którym strumień o niskiej liczbie spienienia znajduje się w dolnej pozycji. Jednak przy czterech takich strumieniach spada całkowita liczba spienienia powstałego strumienia oraz zwiększa się zużycie środka pianotwórczego. Dlatego autorzy przeprowadzili obliczenia porównawcze dla wariantów z dolnym rozlokowaniem trzech i dwóch strumieni piany o niskiej liczbie spienienia. Stwierdzono, że połączenie trzech strumieni piany o niskiej liczbie spienienia zamiast czterech prowadzi do zmniejszenia zasięgu strumienia tylko o $3 \%(0,7 \mathrm{~m})$, ale za to znacznie poprawia całkowitą liczbę spienienia połączonego strumienia. Dalsze zmniejszenie liczby strumieni (do dwóch) skutkuje zauważalnym zmniejszeniem zasięgu skuteczności (5,3\%; 1,1 m) i dodatkowo nie prowadzi do polepszenia spienienia piany w połączonym strumieniu.

W ten sposób, biorąc pod uwagę wpływ strumieni piany o niskiej liczbie spienienia na całkowite spienienie piany połączonego strumienia, najbardziej rozsądne jest zastosowanie trzeciego schematu układu z trzema strumieniami - trzy strumienie powietrzno-mechanicznej piany o niskiej liczbie spieniania podtrzymują od dołu wokół jeden strumień piany o średniej liczbie spienienia.

Wnioski: W niniejszej pracy wykonano badania wzajemnego wpływu strumieni powietrzno-mechanicznych piany o różnych liczbach spienienia. Na podstawie badań teoretycznych i eksperymentalnych określono optymalne rozlokowanie strumieni piany, które pozwalają osiągnąć maksymalny wynik (zasięg) rzutu piany przy minimalnych stratach spienienia. Wyniki przeprowadzonych prac mogą zostać użyte w przyszłości do opracowania eksperymentalnego modelu wytwornicy piany.

Słowa kluczowe: pochyłe strumienie hydrauliczne, model matematyczny, powietrzno-mechaniczna piana, równania, optymalny schemat konstrukcyjny, liczba spienienia, zasięg rzutu piany

Typ artykułu: doniesienie wstępne 


\section{1. Введение}

Анализ тактико-технических возможностей, конструктивных решений и параметров таких пеногенераторов, находящихся на вооружении ГСЧС Украины, свидетельствует об ограниченности сочетания дальности и высоты подачи пены с ее высокой кратностью [1]. Высокая кратность пены обеспечивает не только высокую эффективность тушения пожара, но и малую дальность и высоту подачи струи пены, является результатом очень малой удельной массы и низкой начальной скорости, а следовательно - низкого значения кинетической энергии струи. Малая длина струи пены (6-8 м) требует приближения пожарного к огню, что является не всегда возможным, и всегда - опасно. Снижение кратности пены приводит к увеличению длины струи, но также и к уменьшению эффективности тушения пожара. Учитывая проблему транспортировки пены высокой кратности (К > 200) к месту пожара и высокой вероятности разрушения автоматических установок пожаротушения, легковоспламеняющиеся и горючие жидкости наиболее эффективно тушат воздушно-механической пеной средней кратности $(К=80$ - 100) или используют пленкообразующие пенообразователи на основе фторированных и других поверхностно-активных жидкостей, способных образовывать пленку, которая характеризуется способностью к самовосстановлению после механического разрушения. Оптимальная кратность пен, образованная из таких пенообразователей значительно ниже, а соответственно и дальность струи - выше $[1,2,3]$. Однако широкое использование таких пенообразователей ограничено их высокой стоимостью.

Устранение этих и других недостатков существующих генераторов пены невозможно без обоснования параметров и реализации новых конструктивных решений, которыми ведется применение комбинированного принципа транспортировки пены, что может объединять преимущества конструкций генераторов пены низкой и средней кратности. Соответственно, одним из путей повышения эффективности пожаротушения легковоспламеняющихся и горючих жидкостей и проведения пожарно-спасательных работ является разработка и внедрение установок пеногенераторов, где параллельные струи пены низкой кратности выполняют функцию транспортировки струи средней кратности в зону горения $[4,5]$.

\section{2. Методы}

С целью исследования процесса перемещения в воздухе наклонных гидравлических струй построим математическую модель процесса взаимодействия струй воздушно-механической пены различной кратности. Расчеты будут базироваться на интегрировании уравнений движения материальных точек с учетом сопротивления среды. При взаимодействии одной струи воздушно-механической пены (в дальнейшем просто пены) средней кратности (80-100) и нескольких (четырех) струй пены низкой (8-10) кратности следует также учесть силы, возникающие в результате взаимодействия струй между собой.

С этой целью опишем движение струй, направленных под углом, с помощью уравнений динамики брошенных вверх двух или более абсолютно твердых тел, условно связанных между собой силами. При этом положение тел в пространстве в момент времени $\mathrm{t}$ описывается двумя координатами: $\mathrm{x}=\mathrm{x}(\mathrm{t})$ и $\mathrm{y}=\mathrm{y}(\mathrm{t})$ [4].

На основе анализа литературных данных, приходим к выводу, что наиболее адекватной является квадратичная зависимость силы сопротивления струи при ее трении о воздух. Принимаем коэффициент сопротивления [6]:

$\alpha=\frac{0,316}{\rho D}$

Также примем, что зависимость силы взаимодействия между струями от разности скоростей этих струй характеризуется квадратичной зависимостью.

Сначала смоделируем процесс транспортировки струи средней кратности двумя струями низкой кратности. Получим систему из шести нелинейных дифференциальных уравнений

$$
\begin{aligned}
& \ddot{x}_{0}+\alpha_{0} \cdot \dot{x}_{0} \cdot\left(\dot{x}_{0}^{2}+\dot{y}_{0}^{2}\right)^{1 / 2}+\beta \cdot\left(\dot{x}_{0}-\dot{x}_{1}\right) \cdot\left(\left(\dot{x}_{0}-\dot{x}_{1}\right)^{2}+\left(\dot{y}_{0}-\dot{y}_{1}\right)^{2}\right)^{1 / 2} \\
& +\beta \cdot\left(\dot{x}_{0}-\dot{x}_{2}\right) \cdot\left(\left(\dot{x}_{0}-\dot{x}_{2}\right)^{2}+\left(\dot{y}_{0}-\dot{y}_{2}\right)^{2}\right)^{1 / 2}=0 \\
& \ddot{y}_{0}+\alpha_{0} \cdot \dot{y}_{0} \cdot\left(\dot{x}_{0}^{2}+\dot{y}_{0}^{2}\right)^{1 / 2}+\beta \cdot\left(\dot{y}_{0}-\dot{y}_{1}\right) \cdot\left(\left(\dot{x}_{0}-\dot{x}_{1}\right)^{2}+\left(\dot{y}_{0}-\dot{y}_{1}\right)^{2}\right)^{1 / 2} \\
& +\beta \cdot\left(\dot{y}_{0}-\dot{y}_{2}\right) \cdot\left(\left(\dot{x}_{0}-\dot{x}_{2}\right)^{2}+\left(\dot{y}_{0}-\dot{y}_{2}\right)^{2}\right)^{1 / 2}=-g \\
& \ddot{x}_{1}+\alpha_{1} \cdot \dot{x}_{1} \cdot\left(\dot{x}_{1}^{2}+\dot{y}_{1}^{2}\right)^{1 / 2}+\beta \cdot\left(\dot{x}_{0}-\dot{x}_{1}\right) \cdot\left(\left(\dot{x}_{0}-\dot{x}_{1}\right)^{2}+\left(\dot{y}_{0}-\dot{y}_{1}\right)^{2}\right)^{1 / 2}=0 \\
& \ddot{y}_{1}+\alpha_{1} \cdot \dot{y}_{1} \cdot\left(\dot{x}_{1}^{2}+\dot{y}_{1}^{2}\right)^{1 / 2}+\beta \cdot\left(\dot{y}_{0}-\dot{y}_{1}\right) \cdot\left(\left(\dot{x}_{0}-\dot{x}_{1}\right)^{2}+\left(\dot{y}_{0}-\dot{y}_{1}\right)^{2}\right)^{1 / 2}=-g \\
& \ddot{x}_{2}+\alpha_{2} \cdot \dot{x}_{2} \cdot\left(\dot{x}_{2}^{2}+\dot{y}_{2}^{2}\right)^{1 / 2}+\beta \cdot\left(\dot{x}_{0}-\dot{x}_{2}\right) \cdot\left(\left(\dot{x}_{0}-\dot{x}_{2}\right)^{2}+\left(\dot{y}_{0}-\dot{y}_{2}\right)^{2}\right)^{1 / 2}=0 \\
& \ddot{y}_{2}+\alpha_{2} \cdot \dot{y}_{2} \cdot\left(\dot{x}_{2}^{2}+\dot{y}_{2}^{2}\right)^{1 / 2}+\beta \cdot\left(\dot{y}_{0}-\dot{y}_{2}\right) \cdot\left(\left(\dot{x}_{0}-\dot{x}_{2}\right)^{2}+\left(\dot{y}_{0}-\dot{y}_{2}\right)^{2}\right)^{1 / 2}=-g
\end{aligned}
$$

с начальными условиями:

$$
\begin{aligned}
& x_{0}(0)=0 ; \quad y_{0}(0)=a ; \quad \dot{x}_{0}(0)=v_{00} \cos \Theta_{1} ; \quad \dot{y}_{0}=v_{00} \sin \Theta_{1} \\
& x_{1}(0)=0 ; \quad y_{1}(0)=b ; \quad \dot{x}_{1}(0)=v_{01} \cos \Theta_{1} ; \quad \dot{y}_{1}=v_{01} \sin \Theta_{1} \text {; } \\
& x_{2}(0)=0 ; \quad y_{2}(0)=c ; \quad \dot{x}_{2}(0)=v_{02} \cos \Theta_{2} ; \quad \dot{y}_{2}=v_{02} \sin \Theta_{2} \text {; }
\end{aligned}
$$


Здесь, как и ранее, $v_{0}$ - скорость истечения струи из ствола; $\Theta$ - угол наклона оси ствола к горизонту; $\alpha$ - коэффициент взаимодействия струи с атмосферой; $\beta$ - коэффициент взаимодействия струй между собой. Индексом «0» обозначим струю пены средней кратности, а индексами «1, 2»- струи пены низкой кратности. Константы $a ; b$; $c$ определяются в соответствии со схемами расположения стволов низкой кратности «1, 2, .. n» относительно ствола средней кратности $«$ ».
Также запишем условие, при котором будет происходить взаимодействие между струями:

Если $\left|y_{1}-y_{0}\right|>Y_{1}$ то $\beta=0$
Если $\left|y_{1}-y_{0}\right| \leq Y_{1}$ то $\beta \neq 0$

где $\mathrm{Y}_{1}$ - значение вертикального расстояния между струями и определяется из схемы расположения струй. Такое условие следует записывать для каждой струи низкой кратности.

Постоянные величины, входящие в формулы математической модели представим в таблице 1.

Таблица 1. Постоянные величины, входящие в формулы математической модели

Table 1. Constants included in the formula of the mathematical model

\begin{tabular}{|c|c|}
\hline 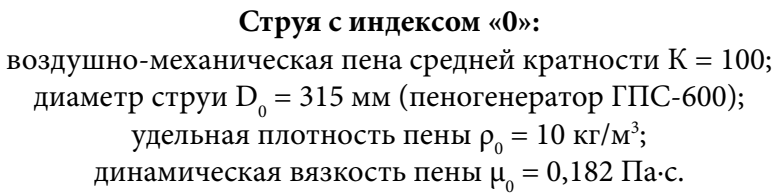 & 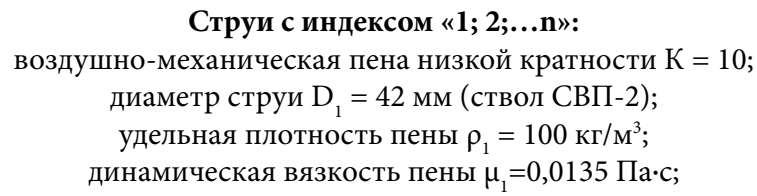 \\
\hline $\begin{array}{c}\text { Stream with the index "0": } \\
\text { air-mechanical foam medium ratio } \mathrm{K}=100 \text {; } \\
\text { stream diameter } \mathrm{D}_{0}=315 \mathrm{~mm}(\text { foam generator GPS-600); } \\
\text { specific density foam } \rho_{0}=10 \mathrm{~kg} / \mathrm{m}^{3} \text {; } \\
\text { the dynamic viscosity of the foam } \mu_{0}=0.182 \mathrm{~Pa} \cdot \mathrm{s} \text {. }\end{array}$ & $\begin{array}{l}\text { Stream with the index "1; } 2 ; \ldots \text { n": } \\
\text { air and mechanical foam of low multiplicity } \mathrm{K}=10 \text {; } \\
\text { stream diameter } \mathrm{D}_{1}=42 \mathrm{~mm}(\text { trunk SAP- } 2) ; \\
\text { the specific density of the foam } \rho_{1}=100 \mathrm{~kg} / \mathrm{m}^{3} \text {; } \\
\text { the dynamic viscosity of the foam } \mu_{1}=0.0135 \mathrm{~Pa} \cdot \mathrm{s} \text {. }\end{array}$ \\
\hline
\end{tabular}

Источник: Собственная разработка.

Source: Own elaboration.

Коэффициенты сопротивления струй при их взаимодействии с воздухом составят:

$\alpha_{1}=0,316 /(10 \cdot 0,3)=0,1053 \mathrm{M}^{-1} ; \alpha_{0}=0,316 /(100 \cdot 0,042)=$ $=0,0752 \mathrm{M}^{-1} ; \Theta_{1}=\Theta_{2}=30^{\circ} \cdot \mathrm{v}_{00}=12,45 \mathrm{M} / \mathrm{c} ; \mathrm{v}_{01}=35,33 \mathrm{M} / \mathrm{c}$ (ориентировочные значения для ствола СВП-2 и пеногенератора ГПС-600).

\section{3. Результаты}

Для определения величины коэффициента $\beta$ от величины относительной скорости струй были проведены предварительные экспериментальные и теоретические исследования на экспериментальной установке, которая состояла из двух воздушно-пенных стандартных стволов СПП-2 и одного стандартного пеногенератора ГПС-600 соединенных между собой. Результаты экспериментальных исследований при различных условиях $\left(\mathrm{p}=5,7,10\right.$ ат. и $\left.\Theta=30^{\circ} ; 45^{\circ}\right)$ были сравнены с результатами решения системы дифференциальных уравнений (2) (после подстановки соответствующих значений) методом Рунге-Кутта в программной среде MATLAB.

Таблица 2. Теоретические исследования дальности полета струй при $\Theta=30^{\circ}$

Table 2. Theoretical studies of the flight range of streams at $\Theta=30^{\circ}$

\begin{tabular}{|c|c|c|c|}
\hline $\left.\mathrm{V}_{00} ; \mathrm{M} / \mathrm{c}\right)$ & 10,53 & 12,45 & 14,88 \\
\hline $\left.\mathrm{V}_{01} ; \mathrm{M} / \mathrm{c}\right)$ & 29,84 & 35,33 & 12,2 \\
\hline $\mathrm{L}_{0} ;(\mathrm{M})$ & 13,67 & 15,59 & 17,68 \\
\hline $\mathrm{L}_{1} ;(\mathrm{M})$ & 13,82 & 15,77 & 0,22 \\
\hline $\left.\mathrm{L}_{1}-\mathrm{L}_{0} ; \mathrm{M}\right)$ & 0,15 & 0,18 & 17,79 \\
\hline$\left(\mathrm{L}_{1}+\mathrm{L}_{0}\right) / 2 ;(\mathrm{M})$ & 13,75 & 15,68 & 18,5 \\
\hline Експер. L (M) & 14,10 & 16,20 & 3,84 \\
\hline$\delta,(\%)$ & 2,48 & 3,21 & \\
\hline$\left.\delta_{\text {cер }}, \%\right)$ & & 3,18 & \\
\hline
\end{tabular}

Источник: Собственная разработка.

Source: Own elaboration. 
Полученные результаты (среднее значение погрешности составляет $3,18 \%$ ) доказывают, что теоретические исследования процесса транспортировки струи воздушно-механической пены средней кратности струей (струями) такой же пены низкой кратности следует проводить по системе нелинейных дифференциальных уравнений (2) с начальными условиями (3) и условиями существования фазы взаимодействия между струями
(3). Коэффициент $\alpha$, характеризующий взаимодействие струй с воздухом, следует определять по формуле (1). При этом $\beta=64$.

Определим оптимальную конструктивную схему расположения струй. Аналогично (2) составляем уравнения для одной центральной струи пены средней кратности и четырех струй пены низкой кратности, взаимодействующих с центральной:

$$
\begin{aligned}
& \ddot{x}_{0}+\alpha_{0} \cdot \dot{x}_{0} \cdot\left(\dot{x}_{0}^{2}+\dot{y}_{0}^{2}\right)^{1 / 2}+\beta_{1} \cdot\left(\dot{x}_{0}-\dot{x}_{1}\right) \cdot\left(\left(\dot{x}_{0}-\dot{x}_{1}\right)^{2}+\left(\dot{y}_{0}-\dot{y}_{1}\right)^{2}\right)^{1 / 2}+ \\
& +\beta_{2} \cdot\left(\dot{x}_{0}-\dot{x}_{2}\right) \cdot\left(\left(\dot{x}_{0}-\dot{x}_{2}\right)^{2}+\left(\dot{y}_{0}-\dot{y}_{2}\right)^{2}\right)^{1 / 2}+\beta_{3} \cdot\left(\dot{x}_{0}-\dot{x}_{3}\right) \cdot\left(\left(\dot{x}_{0}-\dot{x}_{3}\right)^{2}+\left(\dot{y}_{0}-\dot{y}_{3}\right)^{2}\right)^{1 / 2}+ \\
& +\beta_{4} \cdot\left(\dot{x}_{0}-\dot{x}_{4}\right) \cdot\left(\left(\dot{x}_{0}-\dot{x}_{4}\right)^{2}+\left(\dot{y}_{0}-\dot{y}_{4}\right)^{2}\right)^{1 / 2}=0 \\
& \ddot{y}_{0}+\alpha_{0} \cdot \dot{y}_{0} \cdot\left(\dot{x}_{0}^{2}+\dot{y}_{0}^{2}\right)^{1 / 2}+\beta_{1} \cdot\left(\dot{y}_{0}-\dot{y}_{1}\right) \cdot\left(\left(\dot{x}_{0}-\dot{x}_{1}\right)^{2}+\left(\dot{y}_{0}-\dot{y}_{1}\right)^{2}\right)^{1 / 2}+ \\
& +\beta_{2} \cdot\left(\dot{y}_{0}-\dot{y}_{2}\right) \cdot\left(\left(\dot{x}_{0}-\dot{x}_{2}\right)^{2}+\left(\dot{y}_{0}-\dot{y}_{2}\right)^{2}\right)^{1 / 2}+\beta_{3} \cdot\left(\dot{y}_{0}-\dot{y}_{3}\right) \cdot\left(\left(\dot{x}_{0}-\dot{x}_{3}\right)^{2}+\left(\dot{y}_{0}-\dot{y}_{3}\right)^{2}\right)^{1 / 2}+ \\
& +\beta_{4} \cdot\left(\dot{y}_{0}-\dot{y}_{4}\right) \cdot\left(\left(\dot{x}_{0}-\dot{x}_{4}\right)^{2}+\left(\dot{y}_{0}-\dot{y}_{4}\right)^{2}\right)^{1 / 2}=-g \\
& \ddot{x}_{1}+\alpha_{1} \cdot \dot{x}_{1} \cdot\left(\dot{x}_{1}^{2}+\dot{y}_{1}^{2}\right)^{1 / 2}-\beta_{1} \cdot\left(\dot{x}_{0}-\dot{x}_{1}\right) \cdot\left(\left(\dot{x}_{0}-\dot{x}_{1}\right)^{2}+\left(\dot{y}_{0}-\dot{y}_{1}\right)^{2}\right)^{1 / 2}=0 \\
& \ddot{y}_{1}+\alpha_{1} \cdot \dot{y}_{1} \cdot\left(\dot{x}_{1}^{2}+\dot{y}_{1}^{2}\right)^{1 / 2}-\beta_{1} \cdot\left(\dot{y}_{0}-\dot{y}_{1}\right) \cdot\left(\left(\dot{x}_{0}-\dot{x}_{1}\right)^{2}+\left(\dot{y}_{0}-\dot{y}_{1}\right)^{2}\right)^{1 / 2}=-g \\
& \ddot{x}_{2}+\alpha_{2} \cdot \dot{x}_{2} \cdot\left(\dot{x}_{2}^{2}+\dot{y}_{2}^{2}\right)^{1 / 2}-\beta_{2} \cdot\left(\dot{x}_{0}-\dot{x}_{2}\right) \cdot\left(\left(\dot{x}_{0}-\dot{x}_{2}\right)^{2}+\left(\dot{y}_{0}-\dot{y}_{2}\right)^{2}\right)^{1 / 2}=0 \\
& \ddot{y}_{2}+\alpha_{2} \cdot \dot{y}_{2} \cdot\left(\dot{x}_{2}^{2}+\dot{y}_{2}^{2}\right)^{1 / 2}-\beta_{2} \cdot\left(\dot{y}_{0}-\dot{y}_{2}\right) \cdot\left(\left(\dot{x}_{0}-\dot{x}_{2}\right)^{2}+\left(\dot{y}_{0}-\dot{y}_{2}\right)^{2}\right)^{1 / 2}=-g \\
& \ddot{x}_{3}+\alpha_{3} \cdot \dot{x}_{3} \cdot\left(\dot{x}_{3}^{2}+\dot{y}_{3}^{2}\right)^{1 / 2}-\beta_{3} \cdot\left(\dot{x}_{0}-\dot{x}_{3}\right) \cdot\left(\left(\dot{x}_{0}-\dot{x}_{3}\right)^{2}+\left(\dot{y}_{0}-\dot{y}_{3}\right)^{2}\right)^{1 / 2}=0 \\
& \ddot{y}_{3}+\alpha_{3} \cdot \dot{y}_{3} \cdot\left(\dot{x}_{3}^{2}+\dot{y}_{3}^{2}\right)^{1 / 2}-\beta_{3} \cdot\left(\dot{y}_{0}-\dot{y}_{3}\right) \cdot\left(\left(\dot{x}_{0}-\dot{x}_{3}\right)^{2}+\left(\dot{y}_{0}-\dot{y}_{3}\right)^{2}\right)^{1 / 2}=-g \\
& \ddot{x}_{4}+\alpha_{4} \cdot \dot{x}_{4} \cdot\left(\dot{x}_{4}^{2}+\dot{y}_{4}^{2}\right)^{1 / 2}-\beta_{4} \cdot\left(\dot{x}_{0}-\dot{x}_{4}\right) \cdot\left(\left(\dot{x}_{0}-\dot{x}_{4}\right)^{2}+\left(\dot{y}_{0}-\dot{y}_{4}\right)^{2}\right)^{1 / 2}=0 \\
& \ddot{y}_{4}+\alpha_{4} \cdot \dot{y}_{4} \cdot\left(\dot{x}_{4}^{2}+\dot{y}_{4}^{2}\right)^{1 / 2}-\beta_{4} \cdot\left(\dot{y}_{0}-\dot{y}_{4}\right) \cdot\left(\left(\dot{x}_{0}-\dot{x}_{4}\right)^{2}+\left(\dot{y}_{0}-\dot{y}_{4}\right)^{2}\right)^{1 / 2}=-g
\end{aligned}
$$

Выбираем три различных варианта расположения струй (рис. 1):

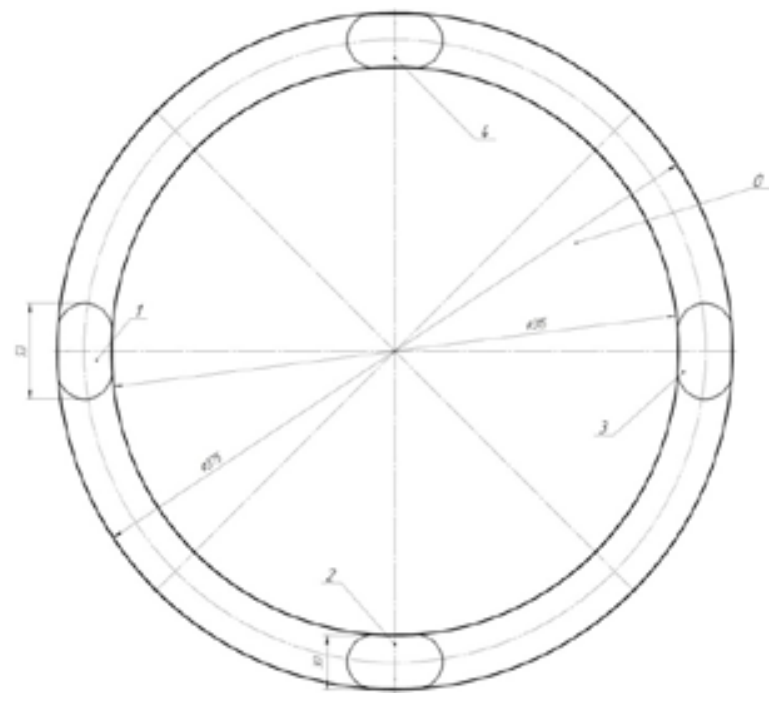

a) / a)

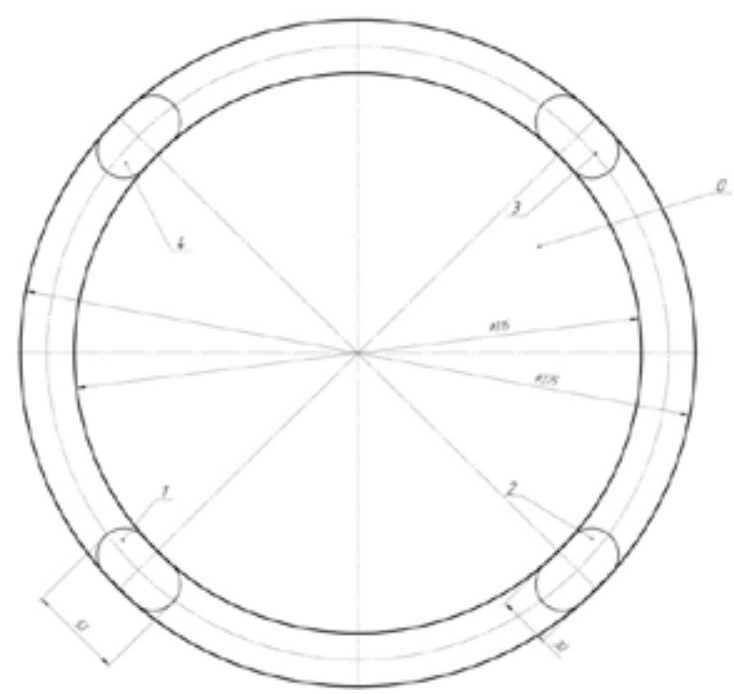

6) / b) 


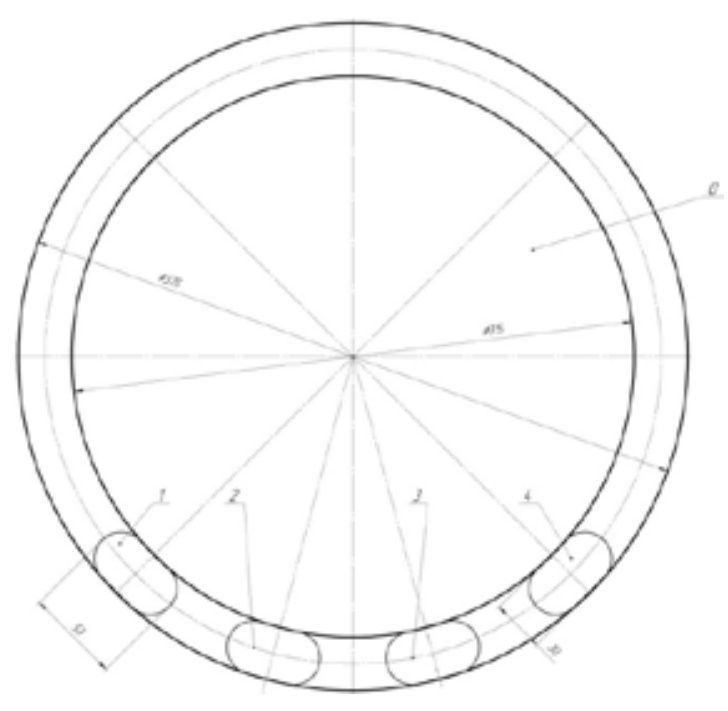

в) / c)

Рис. 1. Варианты конструктивных схем размещения струй:

a) - поддерживающие струи размещены через каждые $90^{\circ}$; б) - поддерживающие струи размещены через каждые $90^{\circ}$ со смещением $45^{\circ}$; в) - поддерживающие струи размещены снизу, где 0 - нулевая струя пены средней кратности; $1,2,3,4$ - поддерживающие струи пены низкой кратности.

Fig. 1. Variants of streams location:

a) - supporting streams placed every $90^{\circ}$; b) - supporting streams placed every $90^{\circ}$ offset $45^{\circ} ; c$ ) - supporting the streams placed below, where 0 - zero stream medium expansion foam; $1,2,3,4$ - supporting streams of low expansion foam.

Источник: Собственная разработка. Source: Own elaboration.

На основе решения системы уравнений был проведен анализ траекторий перемещения струй, режимов совместного движения струй, процессов выравнивания скоростей. Установлено, что наиболее эффективной с точки зрения дальности полета есть третья схема (рис. 2), а эффективность двух предыдущих схем практически одинаковой.

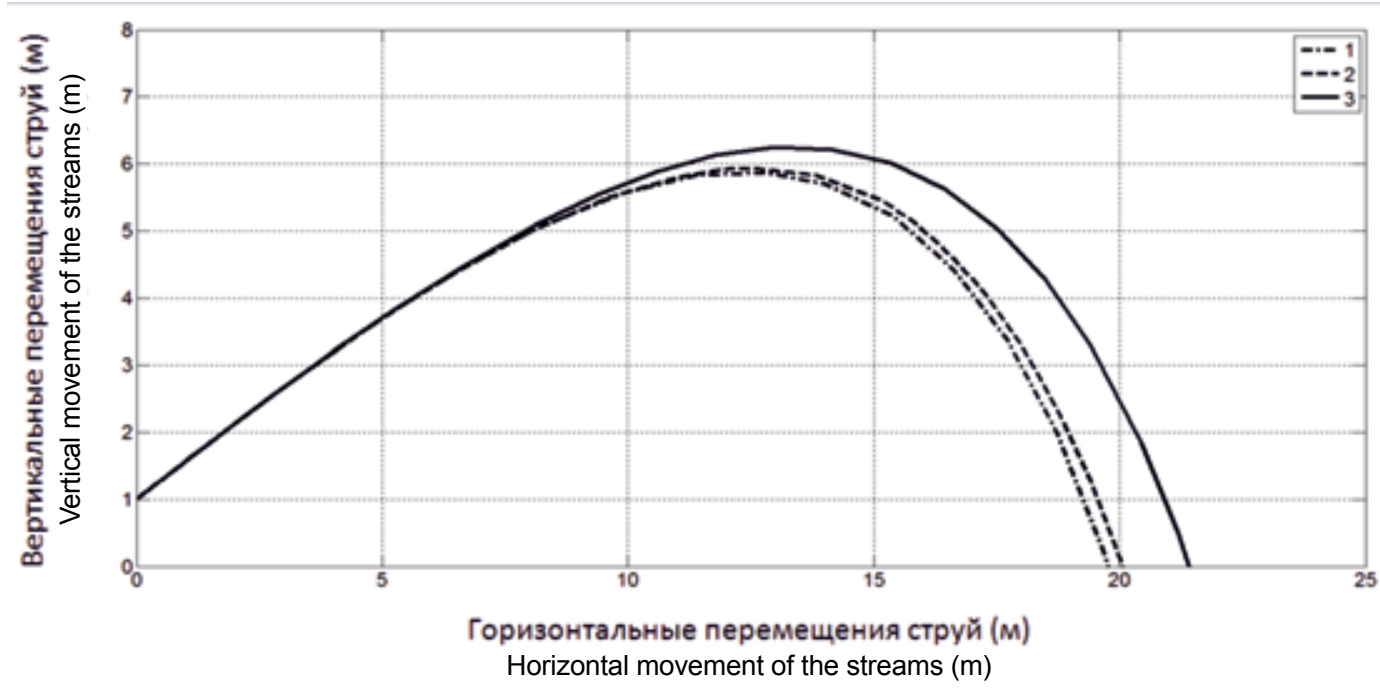

Рис. 2. Траектории перемещений струй пены средней кратности:

1 - использование конструктивной схемы $a$ (рис. 1); 2 - использование конструктивной схемы 6 (рис. 1); 3 - использование конструктивной схемы 8 (рис. 1).

Fig. 2. The path of the medium expansion foam stream:

1 - constructive scheme (a) (Fig. 1); 2 - constructive scheme (b) (Fig. 1); 3 - constructive scheme (c) (Fig. 1).

Источник: Собственная разработка.

Source: Own elaboration.

Необходимо также исследовать влияние ветра на траекторию обобщенной струи. При встречном ветре в систему уравнений (5) введем скорость ветра
V в горизонтальную составляющую скорости струй и получим систему уравнений (6) 


$$
\begin{aligned}
& \ddot{x}_{0}+\alpha_{0} \cdot\left(\dot{x}_{0}+k \cdot V\right) \cdot\left(\left(\dot{x}_{0}+k \cdot V\right)^{2}+\dot{y}_{0}^{2}\right)^{1 / 2}+\beta_{1} \cdot\left(\dot{x}_{0}-\dot{x}_{1}\right) \cdot\left(\left(\dot{x}_{0}-\dot{x}_{1}\right)^{2}+\left(\dot{y}_{0}-\dot{y}_{1}\right)^{2}\right)^{1 / 2}+ \\
& +\beta_{2} \cdot\left(\dot{x}_{0}-\dot{x}_{2}\right) \cdot\left(\left(\dot{x}_{0}-\dot{x}_{2}\right)^{2}+\left(\dot{y}_{0}-\dot{y}_{2}\right)^{2}\right)^{1 / 2}+\beta_{3} \cdot\left(\dot{x}_{0}-\dot{x}_{3}\right) \cdot\left(\left(\dot{x}_{0}-\dot{x}_{3}\right)^{2}+\left(\dot{y}_{0}-\dot{y}_{3}\right)^{2}\right)^{1 / 2}+ \\
& +\beta_{4} \cdot\left(\dot{x}_{0}-\dot{x}_{4}\right) \cdot\left(\left(\dot{x}_{0}-\dot{x}_{4}\right)^{2}+\left(\dot{y}_{0}-\dot{y}_{4}\right)^{2}\right)^{1 / 2}=0 \\
& \ddot{y}_{0}+\alpha_{0} \cdot \dot{y}_{0} \cdot\left(\left(\dot{x}_{0}+k \cdot V\right)^{2}+\dot{y}_{0}^{2}\right)^{1 / 2}+\beta_{1} \cdot\left(\dot{y}_{0}-\dot{y}_{1}\right) \cdot\left(\left(\dot{x}_{0}-\dot{x}_{1}\right)^{2}+\left(\dot{y}_{0}-\dot{y}_{1}\right)^{2}\right)^{1 / 2}+ \\
& +\beta_{2} \cdot\left(\dot{y}_{0}-\dot{y}_{2}\right) \cdot\left(\left(\dot{x}_{0}-\dot{x}_{2}\right)^{2}+\left(\dot{y}_{0}-\dot{y}_{2}\right)^{2}\right)^{1 / 2}+\beta_{3} \cdot\left(\dot{y}_{0}-\dot{y}_{3}\right) \cdot\left(\left(\dot{x}_{0}-\dot{x}_{3}\right)^{2}+\left(\dot{y}_{0}-\dot{y}_{3}\right)^{2}\right)^{1 / 2}+ \\
& +\beta_{4} \cdot\left(\dot{y}_{0}-\dot{y}_{4}\right) \cdot\left(\left(\dot{x}_{0}-\dot{x}_{4}\right)^{2}+\left(\dot{y}_{0}-\dot{y}_{4}\right)^{2}\right)^{1 / 2}=-g \\
& \ddot{x}_{1}+\alpha_{1} \cdot\left(\dot{x}_{1}+k \cdot V\right) \cdot\left(\left(\dot{x}_{1}+k \cdot V\right)^{2}+\dot{y}_{1}^{2}\right)^{1 / 2}-\beta_{1} \cdot\left(\dot{x}_{0}-\dot{x}_{1}\right) \cdot\left(\left(\dot{x}_{0}-\dot{x}_{1}\right)^{2}+\left(\dot{y}_{0}-\dot{y}_{1}\right)^{2}\right)^{1 / 2}=0 \\
& \ddot{y}_{1}+\alpha_{1} \cdot \dot{y}_{1} \cdot\left(\left(\dot{x}_{1}+k \cdot V\right)^{2}+\dot{y}_{1}^{2}\right)^{1 / 2}-\beta_{1} \cdot\left(\dot{y}_{0}-\dot{y}_{1}\right) \cdot\left(\left(\dot{x}_{0}-\dot{x}_{1}\right)^{2}+\left(\dot{y}_{0}-\dot{y}_{1}\right)^{2}\right)^{1 / 2}=-g \\
& \ddot{x}_{2}+\alpha_{2} \cdot\left(\dot{x}_{2}+k \cdot V\right) \cdot\left(\left(\dot{x}_{2}+k \cdot V\right)^{2}+\dot{y}_{2}^{2}\right)^{1 / 2}-\beta_{2} \cdot\left(\dot{x}_{0}-\dot{x}_{2}\right) \cdot\left(\left(\dot{x}_{0}-\dot{x}_{2}\right)^{2}+\left(\dot{y}_{0}-\dot{y}_{2}\right)^{2}\right)^{1 / 2}=0 \\
& \ddot{y}_{2}+\alpha_{2} \cdot \dot{y}_{2} \cdot\left(\left(\dot{x}_{2}+k \cdot V\right)^{2}+\dot{y}_{2}^{2}\right)^{1 / 2}-\beta_{2} \cdot\left(\dot{y}_{0}-\dot{y}_{2}\right) \cdot\left(\left(\dot{x}_{0}-\dot{x}_{2}\right)^{2}+\left(\dot{y}_{0}-\dot{y}_{2}\right)^{2}\right)^{1 / 2}=-g \\
& \ddot{x}_{3}+\alpha_{3} \cdot\left(\dot{x}_{3}+k \cdot V\right) \cdot\left(\left(\dot{x}_{3}+k \cdot V\right)^{2}+\dot{y}_{3}^{2}\right)^{1 / 2}-\beta_{3} \cdot\left(\dot{x}_{0}-\dot{x}_{3}\right) \cdot\left(\left(\dot{x}_{0}-\dot{x}_{3}\right)^{2}+\left(\dot{y}_{0}-\dot{y}_{3}\right)^{2}\right)^{1 / 2}=0 \\
& \ddot{y}_{3}+\alpha_{3} \cdot \dot{y}_{3} \cdot\left(\left(\dot{x}_{3}+k \cdot V\right)^{2}+\dot{y}_{3}^{2}\right)^{1 / 2}-\beta_{3} \cdot\left(\dot{y}_{0}-\dot{y}_{3}\right) \cdot\left(\left(\dot{x}_{0}-\dot{x}_{3}\right)^{2}+\left(\dot{y}_{0}-\dot{y}_{3}\right)^{2}\right)^{1 / 2}=-g \\
& \ddot{x}_{4}+\alpha_{4} \cdot\left(\dot{x}_{4}+k \cdot V\right) \cdot\left(\left(\dot{x}_{4}+k \cdot V\right)^{2}+\dot{y}_{4}^{2}\right)^{1 / 2}-\beta_{4} \cdot\left(\dot{x}_{0}-\dot{x}_{4}\right) \cdot\left(\left(\dot{x}_{0}-\dot{x}_{4}\right)^{2}+\left(\dot{y}_{0}-\dot{y}_{4}\right)^{2}\right)^{1 / 2}=0 \\
& \ddot{y}_{4}+\alpha_{4} \cdot \dot{y}_{4} \cdot\left(\left(\dot{x}_{4}+k \cdot V\right)^{2}+\dot{y}_{4}^{2}\right)^{1 / 2}-\beta_{4} \cdot\left(\dot{y}_{0}-\dot{y}_{4}\right) \cdot\left(\left(\dot{x}_{0}-\dot{x}_{4}\right)^{2}+\left(\dot{y}_{0}-\dot{y}_{4}\right)^{2}\right)^{1 / 2}=-g
\end{aligned}
$$

Решив эту систему для каждого из 3 вариантов размещения струй, получим перемещения и скорости струй при воздействии встречного ветра. Можно сделать вывод, что ветер скоростью 5 м/с является серьезной преградой для пенных струй, однако дальность полета 12 м при 3 варианте все же указывает на возможность эффективного использования установки для тушения пожаров в условиях встречного ветра. Конструктивные схемы 1 и 2 имеют меньшую устойчивость к встречному ветру, а схема 2 (две струи внизу центральной) является эффективнее схемы 1 (одна струя) - 11,5 м и 11 м соответственно.

Для учета действия бокового ветра в систему уравнений 2.13 следует ввести еще одну координату - координату Z, что приведет к росту количества уравнений, а ветер $\mathrm{V}$ теперь будет действовать именно по этой координате. Выполнив эти преобразования получим систему дифференциальных уравнений 7.

$$
\begin{aligned}
& \ddot{x}_{0}+\alpha_{0} \cdot \dot{x}_{0} \cdot\left(\dot{x}_{0}^{2}+\dot{y}_{0}^{2}+\left(\dot{z}_{0}+k \cdot V\right)^{2}\right)^{1 / 2}+\beta_{1} \cdot\left(\dot{x}_{0}-\dot{x}_{1}\right) \cdot\left(\left(\dot{x}_{0}-\dot{x}_{1}\right)^{2}+\left(\dot{y}_{0}-\dot{y}_{1}\right)^{2}+\left(z_{0}-z_{1}\right)^{2}\right)^{1 / 2}+ \\
& +\beta_{2} \cdot\left(\dot{x}_{0}-\dot{x}_{2}\right) \cdot\left(\left(\dot{x}_{0}-\dot{x}_{2}\right)^{2}+\left(\dot{y}_{0}-\dot{y}_{2}\right)^{2}+\left(z_{0}-z_{2}\right)^{2}\right)^{1 / 2}+ \\
& +\beta_{3} \cdot\left(\dot{x}_{0}-\dot{x}_{3}\right) \cdot\left(\left(\dot{x}_{0}-\dot{x}_{3}\right)^{2}+\left(\dot{y}_{0}-\dot{y}_{3}\right)^{2}+\left(z_{0}-z_{3}\right)^{2}\right)^{1 / 2}+ \\
& +\beta_{4} \cdot\left(\dot{x}_{0}-\dot{x}_{4}\right) \cdot\left(\left(\dot{x}_{0}-\dot{x}_{4}\right)^{2}+\left(\dot{y}_{0}-\dot{y}_{4}\right)^{2}+\left(z_{0}-z_{4}\right)^{2}\right)^{1 / 2}=0 \\
& \ddot{y}_{0}+\alpha_{0} \cdot \dot{y}_{0} \cdot\left(\dot{x}_{0}^{2}+\dot{y}_{0}^{2}+\left(\dot{z}_{0}+k \cdot V\right)^{2}\right)^{1 / 2}+\beta_{1} \cdot\left(\dot{y}_{0}-\dot{y}_{1}\right) \cdot\left(\left(\dot{x}_{0}-\dot{x}_{1}\right)^{2}+\left(\dot{y}_{0}-\dot{y}_{1}\right)^{2}+\left(z_{0}-z_{1}\right)^{2}\right)^{1 / 2}+ \\
& +\beta_{2} \cdot\left(\dot{y}_{0}-\dot{y}_{2}\right) \cdot\left(\left(\dot{x}_{0}-\dot{x}_{2}\right)^{2}+\left(\dot{y}_{0}-\dot{y}_{2}\right)^{2}+\left(z_{0}-z_{1}\right)^{2}\right)^{1 / 2}+ \\
& +\beta_{3} \cdot\left(\dot{y}_{0}-\dot{y}_{3}\right) \cdot\left(\left(\dot{x}_{0}-\dot{x}_{3}\right)^{2}+\left(\dot{y}_{0}-\dot{y}_{3}\right)^{2}+\left(z_{0}-z_{1}\right)^{2}\right)^{1 / 2}+ \\
& +\beta_{4} \cdot\left(\dot{y}_{0}-\dot{y}_{4}\right) \cdot\left(\left(\dot{x}_{0}-\dot{x}_{4}\right)^{2}+\left(\dot{y}_{0}-\dot{y}_{4}\right)^{2}+\left(z_{0}-z_{1}\right)^{2}\right)^{1 / 2}=-g \\
& \ddot{z}_{0}+\alpha_{0} \cdot\left(\dot{z}_{0}+k \cdot V\right) \cdot\left(\dot{x}_{0}^{2}+\dot{y}_{0}^{2}+\left(\dot{z}_{0}+k \cdot V\right)^{2}\right)^{1 / 2}+\beta_{1} \cdot\left(\dot{x}_{0}-\dot{x}_{1}\right) \cdot\left(\left(\dot{x}_{0}-\dot{x}_{1}\right)^{2}+\left(\dot{y}_{0}-\dot{y}_{1}\right)^{2}+\left(z_{0}-z_{1}\right)^{2}\right)^{1 / 2}+ \\
& +\beta_{2} \cdot\left(\dot{x}_{0}-\dot{x}_{2}\right) \cdot\left(\left(\dot{x}_{0}-\dot{x}_{2}\right)^{2}+\left(\dot{y}_{0}-\dot{y}_{2}\right)^{2}+\left(z_{0}-z_{2}\right)^{2}\right)^{1 / 2}+ \\
& +\beta_{3} \cdot\left(\dot{x}_{0}-\dot{x}_{3}\right) \cdot\left(\left(\dot{x}_{0}-\dot{x}_{3}\right)^{2}+\left(\dot{y}_{0}-\dot{y}_{3}\right)^{2}+\left(z_{0}-z_{3}\right)^{2}\right)^{1 / 2}+ \\
& +\beta_{4} \cdot\left(\dot{x}_{0}-\dot{x}_{4}\right) \cdot\left(\left(\dot{x}_{0}-\dot{x}_{4}\right)^{2}+\left(\dot{y}_{0}-\dot{y}_{4}\right)^{2}+\left(z_{0}-z_{4}\right)^{2}\right)^{1 / 2}=0 \\
& \ddot{x}_{1}+\alpha_{1} \cdot \dot{x}_{1} \cdot\left(x_{1}^{2}+\dot{y}_{1}^{2}+\left(\dot{z}_{1}+k \cdot V\right)^{2}\right)^{1 / 2}-\beta_{1} \cdot\left(\dot{x}_{0}-\dot{x}_{1}\right) \cdot\left(\left(\dot{x}_{0}-\dot{x}_{1}\right)^{2}+\left(\dot{y}_{0}-\dot{y}_{1}\right)^{2}+\left(z_{0}-z_{1}\right)^{2}\right)^{1 / 2}=0 \\
& \ddot{y}_{1}+\alpha_{1} \cdot \dot{y}_{1} \cdot\left(x_{1}^{2}+\dot{y}_{1}^{2}+\left(\dot{z}_{1}+k \cdot V\right)^{2}\right)^{1 / 2}-\beta_{1} \cdot\left(\dot{x}_{0}-\dot{x}_{1}\right) \cdot\left(\left(\dot{x}_{0}-\dot{x}_{1}\right)^{2}+\left(\dot{y}_{0}-\dot{y}_{1}\right)^{2}+\left(z_{0}-z_{1}\right)^{2}\right)^{1 / 2}=-g \\
& \ddot{z}_{1}+\alpha_{1} \cdot \dot{z}_{1} \cdot\left(x_{1}^{2}+\dot{y}_{1}^{2}+\left(\dot{z}_{1}+k \cdot V\right)^{2}\right)^{1 / 2}-\beta_{1} \cdot\left(\dot{x}_{0}-\dot{x}_{1}\right) \cdot\left(\left(\dot{x}_{0}-\dot{x}_{1}\right)^{2}+\left(\dot{y}_{0}-\dot{y}_{1}\right)^{2}+\left(z_{0}-z_{1}\right)^{2}\right)^{1 / 2}=0
\end{aligned}
$$




$$
\begin{aligned}
& \ddot{x}_{2}+\alpha_{2} \cdot \dot{x}_{2} \cdot\left(x_{2}^{2}+\dot{y}_{2}^{2}+\left(\dot{z}_{2}+k \cdot V\right)^{2}\right)^{1 / 2}-\beta_{2} \cdot\left(\dot{x}_{0}-\dot{x}_{2}\right) \cdot\left(\left(\dot{x}_{0}-\dot{x}_{2}\right)^{2}+\left(\dot{y}_{0}-\dot{y}_{2}\right)^{2}+\left(z_{0}-z_{2}\right)^{2}\right)^{1 / 2}=0 \\
& \ddot{y}_{2}+\alpha_{2} \cdot \dot{y}_{2} \cdot\left(x_{2}^{2}+\dot{y}_{2}^{2}+\left(\dot{z}_{2}+k \cdot V\right)^{2}\right)^{1 / 2}-\beta_{2} \cdot\left(\dot{x}_{0}-\dot{x}_{2}\right) \cdot\left(\left(\dot{x}_{0}-\dot{x}_{2}\right)^{2}+\left(\dot{y}_{0}-\dot{y}_{2}\right)^{2}+\left(z_{0}-z_{2}\right)^{2}\right)^{1 / 2}=-g \\
& \ddot{z}_{2}+\alpha_{2} \cdot\left(\dot{z}_{2}+k \cdot V\right) \cdot\left(x_{2}^{2}+\dot{y}_{2}^{2}+\left(\dot{z}_{2}+k \cdot V\right)^{2}\right)^{1 / 2}-\beta_{2} \cdot\left(\dot{x}_{0}-\dot{x}_{2}\right) \cdot\left(\left(\dot{x}_{0}-\dot{x}_{2}\right)^{2}+\left(\dot{y}_{0}-\dot{y}_{2}\right)^{2}+\left(z_{0}-z_{2}\right)^{2}\right)^{1 / 2}=0 \\
& \ddot{x}_{3}+\alpha_{3} \cdot \dot{x}_{3} \cdot\left(x_{3}^{2}+\dot{y}_{3}^{2}+\left(\dot{z}_{3}+k \cdot V\right)^{2}\right)^{1 / 2}-\beta_{3} \cdot\left(\dot{x}_{0}-\dot{x}_{3}\right) \cdot\left(\left(\dot{x}_{0}-\dot{x}_{3}\right)^{2}+\left(\dot{y}_{0}-\dot{y}_{3}\right)^{2}+\left(z_{0}-z_{3}\right)^{2}\right)^{1 / 2}=0 \\
& \ddot{y}_{3}+\alpha_{3} \cdot \dot{y}_{3} \cdot\left(x_{3}^{2}+\dot{y}_{3}^{2}+\left(\dot{z}_{3}+k \cdot V\right)^{2}\right)^{1 / 2}-\beta_{3} \cdot\left(\dot{x}_{0}-\dot{x}_{3}\right) \cdot\left(\left(\dot{x}_{0}-\dot{x}_{3}\right)^{2}+\left(\dot{y}_{0}-\dot{y}_{3}\right)^{2}+\left(z_{0}-z_{3}\right)^{2}\right)^{1 / 2}=-g \\
& \ddot{z}_{3}+\alpha_{3} \cdot\left(\dot{z}_{3}+k \cdot V\right) \cdot\left(x_{3}^{2}+\dot{y}_{3}^{2}+\left(\dot{z}_{3}+k \cdot V\right)^{2}\right)^{1 / 2}-\beta_{3} \cdot\left(\dot{x}_{0}-\dot{x}_{3}\right) \cdot\left(\left(\dot{x}_{0}-\dot{x}_{3}\right)^{2}+\left(\dot{y}_{0}-\dot{y}_{3}\right)^{2}+\left(z_{0}-z_{3}\right)^{2}\right)^{1 / 2}=0 \\
& \ddot{x}_{4}+\alpha_{4} \cdot \dot{x}_{4} \cdot\left(x_{4}^{2}+\dot{y}_{4}^{2}+\left(\dot{z}_{4}+k \cdot V\right)^{2}\right)^{1 / 2}-\beta_{4} \cdot\left(\dot{x}_{0}-\dot{x}_{4}\right) \cdot\left(\left(\dot{x}_{0}-\dot{x}_{4}\right)^{2}+\left(\dot{y}_{0}-\dot{y}_{4}\right)^{2}+\left(z_{0}-z_{4}\right)^{2}\right)^{1 / 2}=0 \\
& \ddot{y}_{4}+\alpha_{4} \cdot \dot{y}_{4} \cdot\left(x_{4}^{2}+\dot{y}_{4}^{2}+\left(\dot{z}_{4}+k \cdot V\right)^{2}\right)^{1 / 2}-\beta_{4} \cdot\left(\dot{x}_{0}-\dot{x}_{4}\right) \cdot\left(\left(\dot{x}_{0}-\dot{x}_{4}\right)^{2}+\left(\dot{y}_{0}-\dot{y}_{4}\right)^{2}+\left(z_{0}-z_{4}\right)^{2}\right)^{1 / 2}=-g \\
& \ddot{z}_{4}+\alpha_{4} \cdot\left(\dot{z}_{4}+k \cdot V\right) \cdot\left(x_{4}^{2}+\dot{y}_{4}^{2}+\left(\dot{z}_{4}+k \cdot V\right)^{2}\right)^{1 / 2}-\beta_{4} \cdot\left(\dot{x}_{0}-\dot{x}_{4}\right) \cdot\left(\left(\dot{x}_{0}-\dot{x}_{4}\right)^{2}+\left(\dot{y}_{0}-\dot{y}_{4}\right)^{2}+\left(z_{0}-z_{4}\right)^{2}\right)^{1 / 2}=0
\end{aligned}
$$

Как и в предыдущем случае, решениями системы уравнений 7 при начальных условиях будут перемещения и скорости струй при воздействии бокового ветра в координатах X-Y-Z. Можно сделать вывод, что боковой ветер со скоростью 5 м/с сносит струи в сторону (по Z) на величину около 7 м при всех трех вариантах размещения струй. Немного дальше сносит струю пены средней кратности, а струи пены низкой кратности сносятся немного меньше, что вполне закономерно.

Итак, схема с нижним расположением струй пены низкой кратности является эффективной. Но при использовании 4 таких струй происходит снижение общей кратности пены комбинированной струи, также повышается расход пенообразователя. Поэтому мы провели сравнительные расчеты для вариантов с нижним размещением трех и двух струй пены низкой кратности. Было установлено, что использование трех струй пены низкой кратности вместо четырех приводит к уменьшению дальности полета лишь на 3\% (0,7 м), но значительно улучшает общую кратность пены обобщенной струи. Дальнейшее уменьшение числа струй (до двух) приводит к более заметному уменьшению эффективности $(5,3 \% ; 1,1$ м) и при этом не приводит к улучшению кратности пены обобщенной струи.

Итак, учитывая влияние струй пены низкой кратности на общую кратность пены обобщенной струи, наиболее целесообразной является третья схема расположения с тремя струями - три струи воздушномеханической пены низкой кратности, поддерживают снизу по кругу одну струю пены средней кратности.

\section{4. Выводы}

В данной работе было исследовано взаимодействие струй воздушно-механической пены различной кратности. На основе теоретических и экспериментальных исследований определено оптимальное расположение струй пены, которое позволяет достичь максимального значения дальности подачи пены при минимальных потерях кратности. Результаты данного исследования в дальнейшем будут применены для разработки опытного образца пеногенератора.

\section{Литература}

[1] Kovalyshyn V.V., Vasylyeva O.E., Kozyar N.M., Pinne gasinnya, Navch. Posibnyk, Lviv: LDU BGD 2007, 168.

[2] DSTU 3789-89: Pinoutvoryuvachi zagal'nogo pryznachennya dlya gasinnya pozhezh. Zagal'ni tekhinichni vymogy i metody vyprobuvan'.

[3] DSTU 2802-94: Stvoly pozhezhni lafetni kombinovani.

[4] Lushch V.I., Pidvyshchennya efektyvnosti gasinnya pozhezh legkozaymystykh ta goryuchykh rechovyn kombinovanymy pinnymy strumenyamy: Avtoref. dys. ... kand. tekhn. nauk. - L., 2007, 20.
[5] Kovalyshyn V.V., Ulynets' E.M., Grushovinchuk O.V., Kavets'kyy V.V., Doslidzhennya zalezhnosti kratnosti povitryano-mekhanichnoi piny vid geometrychnykh parametriv pinogeneratora, „Naukovyi visnyk UkrNDIPB” Vol. 24 Issue 2, 2011, pp. 74-79.

[6] Ol'shanskiy V.P., Khalipa V.M., Dubovik O.A., Priblizhennye metody rascheta gidravlicheskikh pozharnykh struy, Khar'kov: Mitets' 2004, 113.

Грушовинчук Александр Владимирович, адъюнкт, Львовский государственный университет безопасности жизнедеятельности.

Ковалышин Василий Васильевич, профессор кафедры, доктор технических наук, профессор, Львовский государственный университет безопасности жизнедеятельности.

Кырылив Ярослав Богданович, ведущий научный сотрудник, кандидат технических наук, старший научный сотрудник, Львовский государственный университет безопасности жизнедеятельности. 